\title{
Genetic Determination of the Cytolytic T Lymphocyte Receptor Repertoire
}

\author{
L. A. Sherman ${ }^{1}$
}

\section{A. Introduction}

The immune system has evolved a number of sophisticated molecular mechanisms that assure the maintenance of a highly diverse repertoire of lymphocyte receptors. In view of the vast potential for $\mathrm{V}$ region diversity, the immune system must judiciously choose specificities that are of greatest value for expression in the lymphocyte receptor repertoire. Indeed, the challenge to immunologists no longer lies in explaining the generation of diversity, but rather in understanding the basis for variable region selection at the genomic level and receptor selection at the environmental level. This is, of course, also the ongoing challenge to the immune system.

Our interest in understanding the basis for repertoire selection led us to develop an experimental system that could be used to assess the influence of a variety of genetic, environmental, and developmental factors on expression of the receptor repertoire of cytolytic T lymphocvies (CTL). This entails antigenic stimulation of a large number of CTL clones under limiting dilution culture conditions, followed by identification of the receptor specificity of each clone by fine specificity analysis [1-4]. The murine $\mathrm{H}-2 \mathrm{~K}^{\mathrm{b}}$ antigen is uniquely suited to such an approach owing to the availability of a large number of $\mathrm{H}-2 \mathrm{~K}^{\mathrm{b}}$ mutants that provide a panel of heterologous targets required for fine specificity analysis.

1 Department of Immunology, Scripps Clinic and Research Foundation, La Jolla, California 92037
The results of such an analysis of the $\mathrm{B} 10 . \mathrm{BR}$ response to $\mathrm{H}-2 \mathrm{~K}^{\mathrm{b}}$ is given in Table 1. It is clear that the response against a single alloantigen is highly diverse. In this examination of 78 independently derived primary CTL clones, we observed 34 different receptor specificities, each defined by a different reactivity pattern (RP) on the panel of $\mathrm{K}^{\mathrm{b}}$ mutants. The existence of such a large repertoire, coupled with the limited size of sampling that is feasible, precludes comparison of repertoires on the basis of the presence or absence of particular specificities. Fortunately, superimposed on such diversity is the existence of a small number of highly recurrent specificities. We believe these to be analogous to public idiotypes in antibody responses insofar as they recur frequently among individuals within an inbred strain and, as such, are phenotypic markers for the purpose of repertoire comparisons. Within the B 10.BR response there are three specificities which are recurrent, RP23, RP39, and RP87 [2].

\section{B. The Role of the MHC in Repertoire Determination}

Having established a basis for repertoire comparison, it is possible to assess the contribution of a particular genetic region to repertoire expression by performing a comparable analysis of the genetically distinct murine strain in question. Of particular interest is the role of $\mathrm{MHC}$ in altering repertoire expression. There is a vast literature presenting evidence of the profound effect of MHC on T cell responsiveness; reviewed in [5]. The strategy used by the immune 
Table 1. The B10.BR anti- $\mathrm{K}^{\mathrm{b}} \mathrm{CTL}$ repertoire ${ }^{\mathrm{a}}$

\begin{tabular}{|c|c|c|c|c|c|c|c|c|c|c|c|c|}
\hline & & & & bm 9 & + & + & + & + & - & - & - & - \\
\hline & & & & $\mathrm{bm} 10$ & + & + & - & - & + & + & - & - \\
\hline & & & & $\mathrm{bm} 11$ & + & - & - & + & + & - & - & + \\
\hline $\mathrm{bm}$ & bml 1 & $\mathrm{bm} 3$ & bm4 & & & & & & & & & \\
\hline+ & + & + & + & & 11 & $\cdot$ & $\cdot$ & $\cdot$ & 1 & . & $\cdot$ & $\cdot$ \\
\hline+ & + & - & + & & 1 & 3 & . & $\cdot$ & $\cdot$ & $\cdot$ & $\cdot$ & $\cdot$ \\
\hline+ & - & - & + & & & $\cdot$ & . & $\cdot$ & $\cdot$ & 1 & 1 & $\cdot$ \\
\hline+ & - & + & + & & 1 & 1 & . & 1 & $\cdot$ & $\cdot$ & $\cdot$ & . \\
\hline- & + & + & + & & . & $\cdot$ & . & 1 & $\cdot$ & $\cdot$ & $\cdot$ & 1 \\
\hline- & + & - & + & & 1 & . & 1 & $\cdot$ & $\cdot$ & 3 & $\cdot$ & $\cdot$ \\
\hline- & - & - & + & & . & 5 & 7 & . & $\cdot$ & 12 & 1 & . \\
\hline- & - & + & + & & 1 & $\cdot$ & 1 & . & $\cdot$ & $\cdot$ & $\cdot$ & $\cdot$ \\
\hline+ & + & + & - & & $\cdot$ & $\cdot$ & $\cdot$ & 1 & $\cdot$ & $\cdot$ & & \\
\hline+ & + & - & - & & $\cdot$ & $\cdot$ & $\cdot$ & . & $\cdot$ & · & $\cdot$ & $\cdot$ \\
\hline+ & - & - & - & & $\cdot$ & $\cdot$ & . & · & $\cdot$ & . & 3 & 1 \\
\hline+ & - & + & - & & $\cdot$ & $\cdot$ & $\cdot$ & 1 & $\cdot$ & 1 & $\cdot$ & 2 \\
\hline- & + & + & - & & 1 & $\cdot$ & $\cdot$ & . & $\cdot$ & • & $\cdot$ & $\cdot$ \\
\hline- & + & - & - & & $\cdot$ & $\cdot$ & 1 & $\cdot$ & $\cdot$ & $\cdot$ & $\cdot$ & $\cdot$ \\
\hline- & - & - & - & & $\cdot$ & 1 & 2 & . & $\cdot$ & . & 3 & $\cdot$ \\
\hline- & - & + & - & & $\cdot$ & $\cdot$ & 1 & 4 & $\cdot$ & $\cdot$ & · & 1 \\
\hline
\end{tabular}

a Each number represents the total number of clones observed which gave the indicated reactivity pattern (RP) [2]

system to focus on cellular presented antigens is the requirement for MHC-restricted recognition. The particular alleles recognized are determined by the MHC antigens expressed by the thymus $[5,6]$. Is this predilection for "self" reflected within the receptor repertoire? Other types of MHCdirected effects on $\mathrm{T}$ cell responsiveness include tolerance and immune response gene (IR) phenomenon. Unlike restriction phenotype, which is a form of positive selection, both IR and tolerance are manifested as the loss of responsiveness associated with a particular MHC haplotype and therefore would be reflected as a negative effect on repertoire.

Figure 1 lists all such recurrent specificities that appear in the $\mathrm{H}-2 \mathrm{~K}^{\mathrm{b}}$-specific response of B10.BR and B10.D2. It may be seen that each of these MHC-disparate, yet otherwise genetically identical strains expresses different recurrent specificities. In order to determine if these differences are reflective of negative or positive $\mathrm{MHC}$ directed influences we next examined the repertoire of (B10.BR $\times$ B10.D2) $F_{1}$ hybrids. These results (Fig. 1) indicate that some specificities, such as RP47, are maintained within an $F_{1}$ environment, whereas others, such as RP87 and RP39, are represented at a much reduced level. Therefore, both positive and negative MHC influences are apparent.

This discussion has made an important assumption which is not necessarily warranted. Specifically, we assumed that all MHC effects are extracellular in origin and not determined by the MHC genotype of the $\mathrm{T}$ cell, but rather by the MHC phenotype of the environment. If this assumption is correct, we would anticipate that, having matured in the identical environment, cells of either $\mathrm{H}-2^{\mathrm{d}}$ or $\mathrm{H}-2^{\mathrm{k}}$ origin should be capable of expressing the identical repertoire. To test this prediction, we constructed double chimeras by repopulating lethally irradiated (B10.D2 $\times$ B10.BR) $\quad F_{1}$ mice with a mixture of fetal liver stem cells of B10.BR and B10.D2 origin. At 8 weeks after reconstruction, individual mice were killed and their spleen cells divided in half and treated with either anti-H-2 $2^{\mathrm{k}}$ or anti-H-2 ${ }^{\mathrm{d}}$ antibodies in the presence of complement. This procedure provides lymphocytes of different MHC genotype that have matured in an identical environment. Each repertoire was independently assessed (Fig. 2). Unlike the results already described, de- 

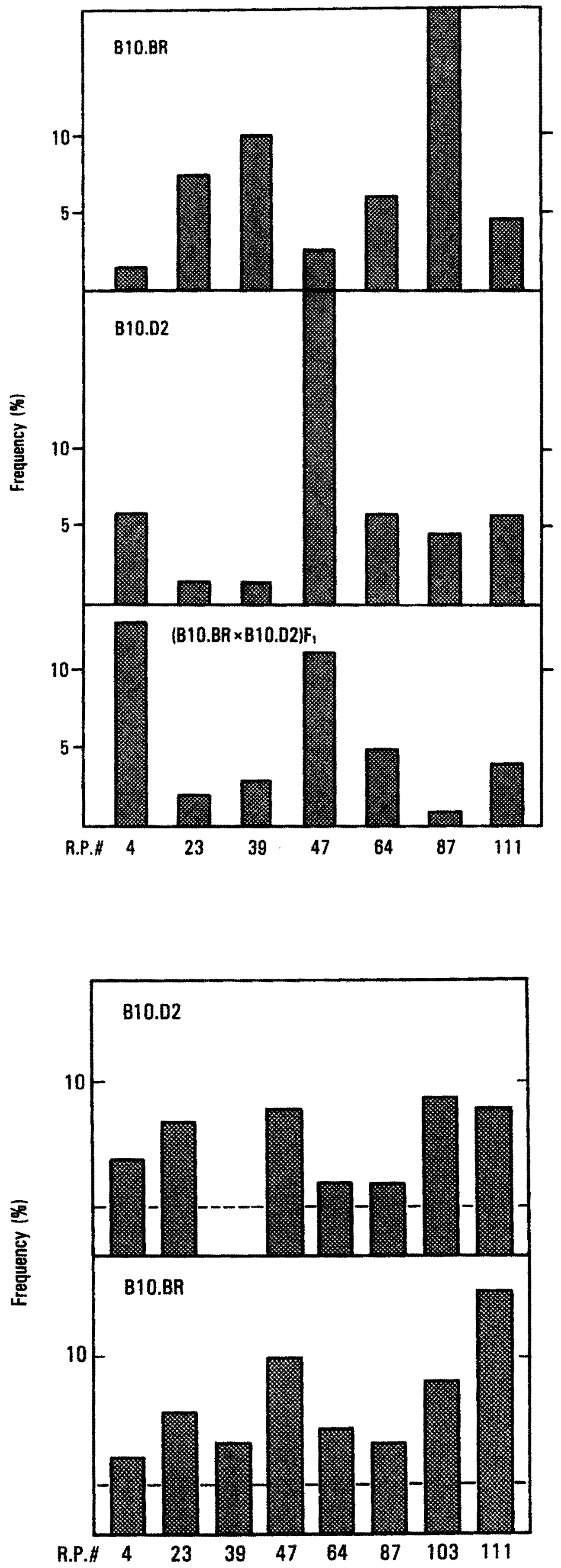

Fig. 1. Comparison of recurrent specificities exhibited by MHC congeneic strains and their $F_{1}$ hybrids. Data represent analysis of over 20 individually analyzed mice [2]
Fig. 2. Analysis of the repertoire of B10.D2 and B10.BR cells obtained from double-radiation chimeras 


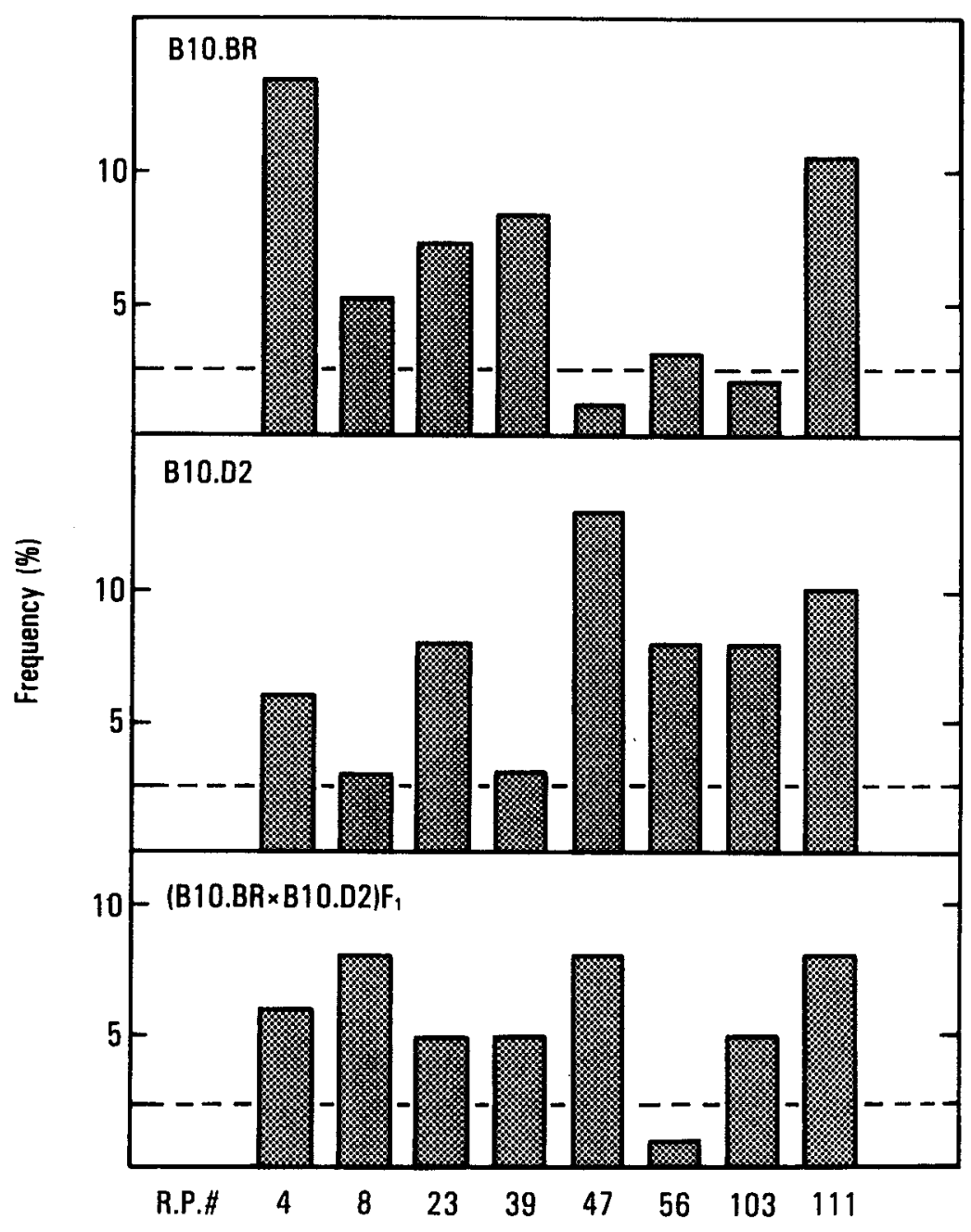

Fig. 3. Comparison of recurrent specificities exhibited by $10-$ to $14-$ day-old neonates [3]

spite their MHC differences, the repertoires of both cell types were very similar. Seven of eight recurrent specificities were comparably expressed by both cell types. Therefore, the effect of MHC observed in this system is essentially environmental in origin.

Another type of environmental influence which could be present in the splenic repertoire would be the effect of continual exposure to antigen in the context of selfMHC. This could yield a repertoire skewed toward self-recognition and would therefore be perceived as an MHC-linked environmental influence. To assess the contribution of post-thymic effects on repertoire we investigated the neonatal CTL response [3]. The rationale for this study is that neonates have experienced far less exposure to antigen. Indeed, as shown in Fig. 3, B10.D2 and $\mathrm{B} 10 . \mathrm{BR}$ neonates demonstrate a significant number of repertoire similarities. In addition, it is clear that some recurrent specificities are unique to each strain and, most significantly, RP39 is unique to B10.BR in both the neonate and adult, whereas RP47 is unique to B10.D2. These $\mathrm{RP}$, therefore, represent potential examples of positive selection in the thymus. Finally, it should be apparent that, unlike the adult $F_{1}$ hybrid, in most cases, neonates express the admixture of both parental (neonatal) repertoires. This indicates that coexpression of most parental specificities is permitted in an $F_{1}$ environment and, therefore, few effects of tolerance are evident inthe neonatal $F_{1}$ repertoire as judged by the $H-2 K^{b}$ specificity repertoire. This again suggests that many of the repertoire features characteristic of the adult may be the result of postthymic effects.

\section{The Role of IgH-Linked Genes in Repertoire Determination}

Having observed a profound influence of MHC on the expressed T cell repertoire, we 


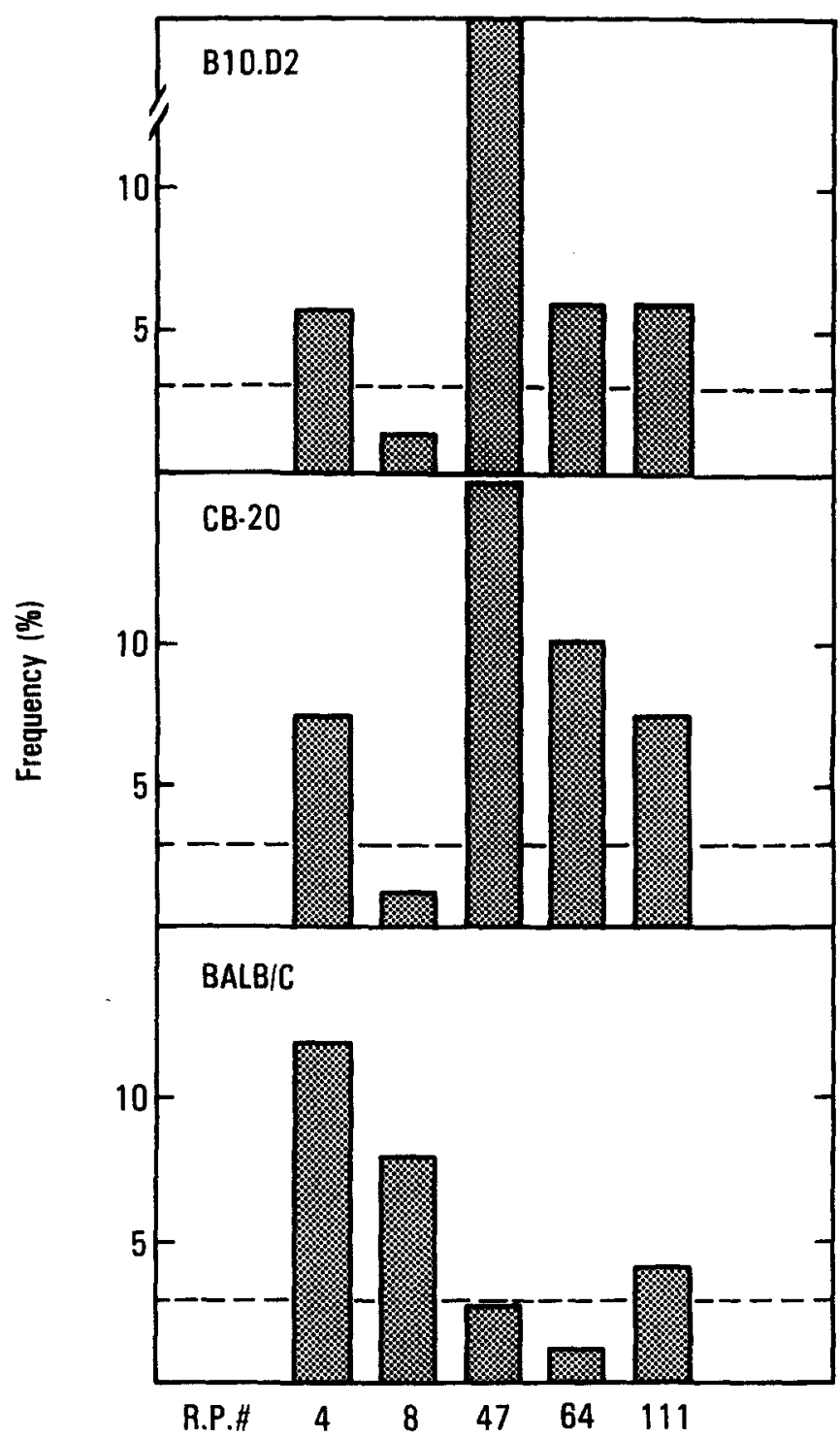

Fig. 4. Recurrent specificities representative of MHC-identical mice with different IgH allotypes. B10.D2 and CB-20 both express Igh ${ }^{\text {b }}$. $\mathrm{BALB} / \mathrm{c}$ expresses $\operatorname{Igh}^{\mathrm{a}}$

next wished to determine if other polymorphic genetic loci affect receptor expression. Inasmuch as numerous laboratories have reported the presence of Igh-linked idiotypic determinants on $\mathrm{T}$ cell products, this polymorphic region is an obvious candidate.

But what about other polymorphic loci? In order simultaneously to screen the effect of numerous polymorphic genes on $T$ repertoire expression, we compared the $\mathrm{H}-2 \mathrm{~K}^{\mathrm{b}}$ specific responses of two strains which share both $\mathrm{MHC}$ and Igh yet are otherwise of independent origin, $\mathrm{CB}-20$ and B10.D2 [4]. Both strains are $\mathrm{H}-2^{\mathrm{d}}, \mathrm{Igh}^{\mathrm{b}}$ yet $\mathrm{CB}-20$ is of $\mathrm{BALB} / \mathrm{C}$ origin and therefore broadly differs from B10.D2. The results of this analysis are presented in Fig. 4. It may be seen that the same recurrent specificities are observed in both repertoires. This implies the polymorphic differences between these two genetic backgrounds other than $\mathrm{MHC}$ and IgH are, in general, of little consequence to the expression of the $\mathrm{H}-2 \mathrm{~K}^{\mathrm{b}}$ specificity repertoire. In contrast, the allotype-congeneic strains CB-20 (Igh $\left.{ }^{b}\right)$ and $\mathrm{BALB} / \mathrm{c}\left(\operatorname{Igh}^{2}\right)$ reveal a large number of significant repertoire differences. Most prominent is the decreased expression of RP47 and RP64 and the increased expression of RP8 in BALB/c. Therefore, allotype-linked genes profoundly influence the expressed $\mathrm{T}$ cell repertoire.

A variety of mechanisms could account for Igh-linked repertoire differences. To help narrow down the possibilities, it was necessary to determine if variable or constant region-linked genes were involved. To address this question, we took advantage of two strains in which recombination within the Igh locus has occurred. BAB-14 has the same variable region genes as $\mathrm{BALB} / \mathrm{c}$ $\left(I g h-V^{a}\right)$ yet the constant region is $I g h-C^{b}$. CBB 7904 is the reciprocal recombinant, Igh- $V^{b}-C^{a}$. The frequency of RP8, RP47, and RP64 in these strains is given in Fig. 5. These results suggest the levels of expression of these particular specificities are determined primarily by Igh-V-linked genes.

Although our data are consistent with the possibility that $T$ cell receptor $V$ regions are linked to Igh- $V$, in view of the susceptibility of repertoire to environmental regulatory events it is also possible to explain these data as a result of immunoregulatory selection by immunoglobulin variable regions $[8,9]$. We are currently distinguishing between these two possibilities by examining the repertoire of allotype-different cells originating from double chimeras in which cells of Igh- $\mathrm{V}^{\mathrm{a}}$ and Igh- $\mathrm{V}^{\mathrm{b}}$ type are permitted to mature together.

In conclusion, we have identified two genetic regions that influence expression of the $T$ cell receptor repertoire, MHC- and Igh-V-linked genes. It is important to note that our methods assess only the contributions of polymorphic genetic loci. Accordingly, we would predict that any other genetic region that participates in receptor expression is either nonpolymorphic be- 


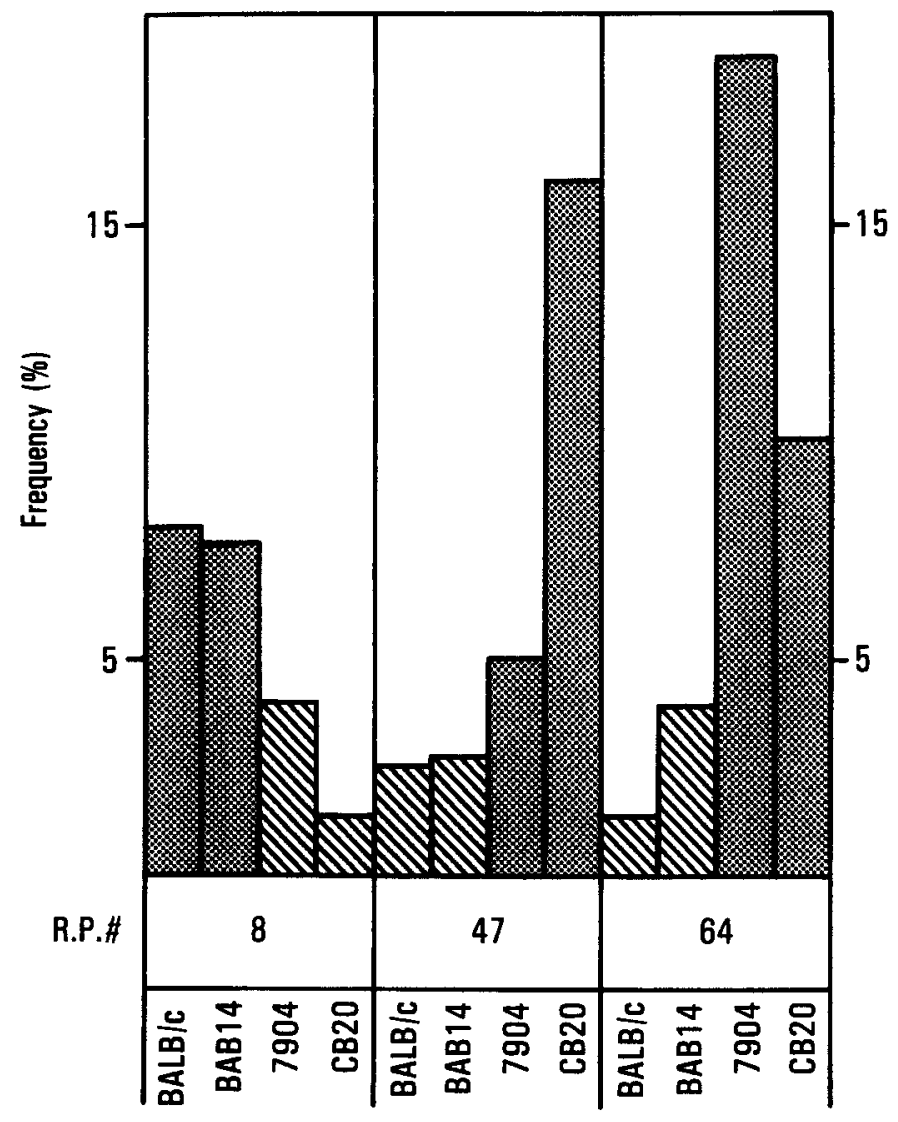

Fig. 5. The effect of recombination within Igh on expression of recurrent specificities tween the BALB/c and B10 backgrounds, or its polymorphism is of limited consequence to repertoire expression.

Acknowledgments. This work was supported by National Institutes of Health Grant AI-20026 and grant IMM-324 from the American Cancer Society.

\section{References}

1. Sherman LA (1980) Dissecting the B10.D2 anti-H-2K ${ }^{b}$ receptor repertoire. J Exp Med 151: 1386

2. Sherman LA (1982) The influence of the major histocompatibility complex on the repertoire of allospecific cytolytic T lymphocytes. $\mathrm{J}$ Exp Med 155:380

3. Sherman LA (1982) Genetic and regulatory contributions of the major histocompatibility complex to the developing cytolytic $\mathrm{T}$ lymphocyte repertoire. J Immunol 128: 1849

4. Sherman LA (1982) Genetic linkage of the cytolytic $T$ lymphocyte repertoire and immunoglobulin heavy chain genes. J Exp Med $156: 294$

5. Zinkernagel RM, Doherty PC (1979) MHC restricted cytotoxic T cells. Adv 27:51

6. Fink PJ, Bevan MJ (1978) H-2 antigens of the thymus determine lymphocyte specificity. J Exp Med 148:766

7. Schwartz R-H (1978) A clonal deletion mode for IR gene control of the immune response. Scand J Immunol 7:3

8. Rajewsky K, Takemori T (1983) Genetics, expression and function of idiotypes. Ann Rev Immunol 1:569

9. Miller JFAP, Morahan G, Walker ID (1983) T cell antigen receptors: fact and artifact. Immunol Today 4:141 